\title{
CORRECTION
}

\section{Author Correction: H3K14me3 genomic distributions and its regulation by KDM4 family demethylases}

Bin Zhao ${ }^{1}{ }^{1}$, Wenqi $\mathrm{Xu}^{1}$, Bowen Rong ${ }^{1}$, Guoyu Chen ${ }^{2}$, Xuanjia Ye ${ }^{1}$, Ruofei Dai ${ }^{1}$, Wenjing Li ${ }^{1}$, Jiajia Chen ${ }^{3}$, Jiajun Cai ${ }^{4}$, Lei Song ${ }^{5}$, Zhao-Qing Luo ${ }^{5}{ }^{5}$, Rong Zeng ${ }^{6}$, Yang Shi ${ }^{7}$, Jing-Dong J. Han ${ }^{2}$ and Fei Lan ${ }^{1}$

Cell Research (2019) 29:90; https://doi.org/10.1038/s41422-018-0118-3

Correction to: Cell Research (2018) 28:1; https://doi.org/10.1038/ s41422-018-0095-6; published online 18 October 2018.

We apologize for an error introduced during format conversion in the paper published online on 18 October 2018. The resolution of the MALDI-TOF result in Fig. 1e underwent an unexpected reduction when transformed from Powerpoint format. The corrected Fig. 1e is provided below.

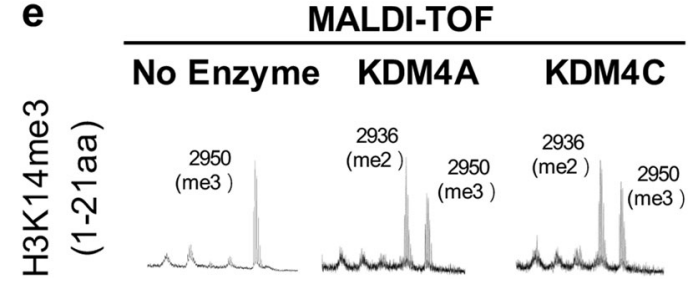

Fig. 1 Corrected Fig. 1e

\footnotetext{
${ }^{1}$ Liver Cancer Institute, Zhongshan Hospital, Fudan University, Key Laboratory of Carcinogenesis and Cancer Invasion, Ministry of Education, Key Laboratory of Epigenetics and

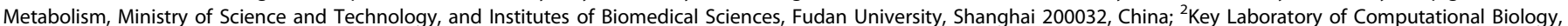

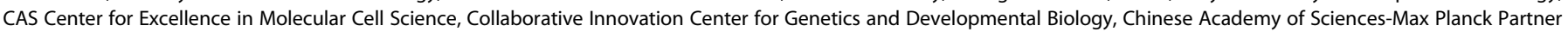

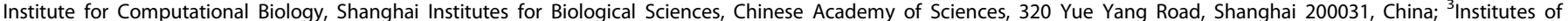

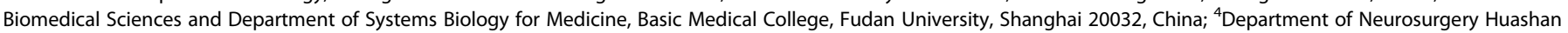

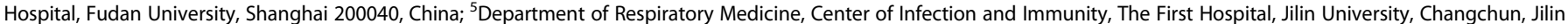

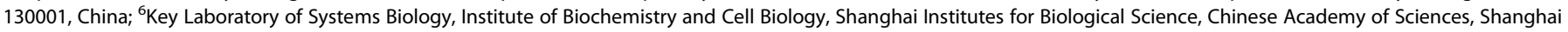
200031, China and ${ }^{7}$ Newborn Medicine Division, Boston Children's Hospital and Department of Cell Biology, Harvard Medical School, Boston, MA 02115, USA

Correspondence: Fei Lan (fei_lan@fudan.edu.cn)

These authors contributed equally: Wenqi Xu, Bowen Rong, Guoyu Chen
}

Published online: 17 December 2018 